How special relativity determines the signs of the nonrelativistic Coulomb and Newtonian forces

S. Deser

Citation: 73, (2005); doi: 10.1119/1.1898503

View online: http://dx.doi.org/10.1119/1.1898503

View Table of Contents: http://aapt.scitation.org/toc/ajp/73/8

Published by the American Association of Physics Teachers 


\title{
How special relativity determines the signs of the nonrelativistic Coulomb and Newtonian forces
}

\author{
S. Deser ${ }^{\mathrm{a}}$ \\ Department of Physics, Brandeis University, Waltham, Massachusetts 02454
}

(Received 4 November 2004; accepted 25 February 2005)

\begin{abstract}
We show that the empirical signs of the fundamental static Coulomb/Newton forces are dictated by the seemingly unrelated requirement that the photons/gravitons in the respective underlying Maxwell/Einstein physics be stable. This linkage, which is imposed by special relativity, is manifested upon decomposing the corresponding fields and sources in a gauge-invariant way, and without appeal to static limits. The signs of these free field excitation energies determine those of the instantaneous forces between sources; opposite Coulomb/Newton signs are direct consequences of the Maxwell/Einstein free excitations' odd/even spins. (C) 2005 American Association of Physics Teachers.
\end{abstract}

[DOI: $10.1119 / 1.1898503]$

\section{INTRODUCTION}

One of the less heralded triumphs of special relativity (SR) is that it determines the signs of the interactions between sources according to the spins of their mediating fields. In contrast, these signs are arbitrary in nonrelativistic physics: the observed Coulomb/Newtonian repulsion/attraction must be put in by hand. SR bans instantaneous action-at-a-distance in favor of mediating, and necessarily dynamical, fields. The resulting Maxwell/Einstein framework then predicts these static properties. As we will see, the (lightlike) free excitations' energy signs are rigidly (if not obviously) linked to those of the nonrelativistic regime's source-source interactions, where these (classical) "photons" and "gravitons" otherwise play no role at all. The interactions' signs are determined by the odd/even spins of the mediating fields.

We will carry out the derivations both by a simple static limit shortcut and by a (more elaborate) gauge-invariant procedure, where time independence is not invoked. Appendix A extends our results to the more general, but less physical, systems of arbitrary spin and to form fields; Appendix B provides a quick covariant (but more technical) exposition of the phenomenon.

\section{MEDIATING FIELDS}

Nonrelativistically, action-at-a-distance is translated into a local field framework by defining a scalar potential field $\phi$ with the action

$$
I_{\mathrm{nr}}[\phi ; \rho]=\frac{\epsilon}{2} \int d^{d} x \phi\left(-\nabla^{2}\right) \phi+\int d^{d} x \rho \phi,
$$

which is to be added to the free particle actions. Here, $\epsilon$ $= \pm 1$ is a sign factor, $\rho$ is the particle density, and $d$ is the space dimensionality, which does not affect the analysis. [We also could include a parameter $m^{2}$ to cover both infinite ( $m$ $=0)$ and finite $(m \neq 0)$ range forces by using the (positive) Yukawa operator $\left(-\nabla^{2}+m^{2}\right)$.] The sign of the force between particles is obtained after a field-redefinition, $\phi=\widetilde{\phi}$ $+\epsilon G \rho$, where $-G$ is the usual Coulomb Green function,

$$
\nabla^{2} G\left(\mathbf{r}-\mathbf{r}^{\prime}\right)=\delta^{d}\left(\mathbf{r}-\mathbf{r}^{\prime}\right) .
$$

Equation (1) is recast in terms of $G$ as

$$
I_{\mathrm{nr}}[\widetilde{\phi} ; \rho]=\frac{\epsilon}{2} \int d^{d} x \widetilde{\phi}\left(-\nabla^{2}\right) \widetilde{\phi}+\frac{\epsilon}{2} \int d^{d} x \rho G \rho .
$$

The free $\widetilde{\phi}$-field obeys the Laplace equation and simply decouples; the net interaction resides entirely in the second term of Eq. (3) whose sign depends only on that of the freefield action. Because this sign, $\epsilon$, is arbitrary, the choice of attraction/repulsion $(\epsilon=\mp)$ has to be inserted by hand. [To check this sign correlation, write $\rho=q_{1} \delta^{d}\left(\mathbf{r}-\mathbf{r}_{1}\right)+q_{2} \delta^{d}(\mathbf{r}$ $-\mathbf{r}_{2}$ ), that is, as a sum of point sources, keep the cross terms, remembering that a positive potential term in an action, $\int(T-V)$, corresponds to attractive, negative, $V$.]

The first example of how SR determines everything is the (nongauge) scalar field itself. We must first promote the Laplacian to the wave operator, $\nabla^{2} \rightarrow \square \equiv \nabla^{2}-\partial^{2} / \partial(c t)^{2}$; the free field part of the action (1) then becomes after an integration by parts,

$$
I_{s}=\frac{1}{2} \int d^{d} x d t\left\{\left[\dot{\phi}^{2}+\phi \nabla^{2} \phi\right]+2 \rho \phi\right\},
$$

where $c=1$ henceforth. The relative sign in Eq. (4) is thus fixed by SR; its overall sign ensures that the scalar field's newly acquired free excitation mode has positive energy with respect to the usual convention for a free particle's, $I_{p}$ $=(m / 2) \int d t \dot{x}^{2}$. Otherwise, there would be no stable ground state because as the particles radiate the field away, they would gain energy! So a scalar's $\epsilon$ sign is necessarily negative, corresponding to attraction between like sources. To summarize, SR here forced the sign of the static, "action-ata-distance" part of the action by the seemingly remote twostep requirements of "covariantization," $\nabla^{2} \rightarrow \square$, and of positive kinetic energy of the resulting free field excitations.

\section{MAXWELL}

We now come to the first physical example, Coulomb repulsion. The Maxwell field's action is 


$$
\begin{aligned}
I_{M}\left[A_{\mu}, j^{\mu}\right] \equiv & -\frac{1}{4} \int d t d^{d} x\left[F_{\mu \nu} F^{\mu \nu}-4 A_{\mu} j^{\mu}\right] \\
= & \int d t d^{d} x\left[\frac{1}{2}\left(\left(\nabla A_{0}-\dot{\mathbf{A}}\right)^{2}-(\nabla \times \mathbf{A})^{2}\right)+\mathbf{j} \cdot \mathbf{A}\right. \\
& \left.+j^{0} A_{0}\right]
\end{aligned}
$$

in terms of $F_{\mu \nu}=\partial_{\mu} A_{\nu}-\partial_{\nu} A_{\mu}$, with signature $(-,+++)$. The static, Coulomb, force concerns only $j^{0}$, and does not involve the vector potential $\mathbf{A}$, although $\mathbf{A}$ alone determines the overall sign of Eq. (5) and thereby of the force. That is, the sign of the action is again fixed by the positivity of the kinetic term in $I_{M}$ which describes the pure "photon" excitations,

$$
\begin{aligned}
I_{M}= & +\int d t d^{d} x\left\{\frac{1}{2}\left[\dot{\mathbf{A}}^{2}-(\boldsymbol{\nabla} \times \mathbf{A})^{2}\right]+\frac{1}{2}\left(\nabla A_{0}\right)^{2}+j^{0} A_{0}\right. \\
& \left.+\mathbf{j} \cdot \mathbf{A}-\boldsymbol{\nabla} A_{0} \cdot \dot{\mathbf{A}}\right\} .
\end{aligned}
$$

Indeed, $A_{0}$ is not dynamical at all, but an auxiliary variable that enforces Gauss's law. The time-independent, Coulomb, part of Eq. (6) is then

$$
I_{M} \rightarrow \int d t d^{d} x\left[-\frac{1}{2} A_{0} \nabla^{2} A_{0}+j^{0} A_{0}\right] .
$$

We also may follow the scalar field's redefinition procedure used to reach Eq. (3): Let $A_{0} \rightarrow \widetilde{A}_{0}+G\left(j^{0}+\boldsymbol{\nabla} \cdot \dot{\mathbf{A}}\right)$, which again leads to a decoupled $\widetilde{A}_{0}$ field and the "residual" repulsion

$$
I \rightarrow \frac{1}{2} \int d^{d} x j^{0} G j^{0} .
$$

The above discussion has the drawback that it is not entirely gauge invariant, and makes the implicit gauge choice $\dot{\mathbf{A}}^{L}=0$ to obtain Eq. (7); also the nonrelativistic limit is not needed. Potentials can be eliminated altogether by using the continuity equation, $\boldsymbol{\nabla} \cdot \mathbf{j}+\partial_{0} j^{0}=0$ (forced by gauge invariance) to remove the current's divergence in terms of $j^{0}$, and hence to write the coupling as $\int j^{0} \mathcal{E}$, where the scalar $\mathcal{E}$ is essentially the divergence of the electric field $\mathbf{E} \equiv \boldsymbol{\nabla} A_{0}-\dot{\mathbf{A}}$, namely $\mathcal{E} \equiv G \boldsymbol{\nabla} \cdot \mathbf{E}$. For this purpose, we resort to the orthogonal decomposition of any vector field,

$$
\mathbf{A}=\mathbf{A}^{T}+\mathbf{A}^{L}, \quad \boldsymbol{\nabla} \cdot \mathbf{A}^{T}=0=\nabla \times \mathbf{A}^{L}, \quad \int d^{d} x \mathbf{A}^{T} \cdot \mathbf{B}^{L}=0 ;
$$

the orthogonality between any two $T$ and $L$ vectors expressed in the last relation is especially important. This partition of a vector field is the Fourier transform of the simple momentum space algebraic decomposition,

$$
\begin{aligned}
& \mathbf{A}(\mathbf{k})=-\hat{\mathbf{k}} \times(\hat{\mathbf{k}} \times \mathbf{A})+\hat{\mathbf{k}}(\hat{\mathbf{k}} \cdot \mathbf{A}) \equiv \mathbf{A}^{T}(\mathbf{k})+\mathbf{A}^{L}(\mathbf{k}), \\
& \mathbf{A}^{T}(\mathbf{k}) \cdot \mathbf{B}^{L}(\mathbf{k})=0,
\end{aligned}
$$

in terms of some unit vector $\hat{\mathbf{k}}$ (using $d=3$ notation). The part of the action (6) involving $j^{0}$ is the sum of the coupling and kinetic Maxwell terms:

$$
I_{M} \rightarrow \int d t d^{d} x\left[-\frac{1}{2} \mathcal{E} \nabla^{2} \mathcal{E}+j^{0} \mathcal{E}\right] .
$$

[We need not belabor the now familiar drill, defining $\mathcal{E} \rightarrow \widetilde{\mathcal{E}}$ $+G j^{0}$, etc.] Both Eqs. (7) and (9) lead to Coulomb repulsion, as is clear because the "potentials" $A_{0}$ or $\mathcal{E}$ appear with opposite $\epsilon$-sign to the (attractive) scalar potentials. [Parenthetically, another fundamental by-product of SR is that Maxwell's (and Einstein's) equations contain additional static information that is unavailable to nonrelativistic descriptions: the time-constancy of the electric charge and gravitational mass. ${ }^{1}$ These conservation laws follow from the exclusion of monopole radiation in gauge theories, whereas nothing forbids time-varying "charges" nonrelativistically or for scalar fields.]

Finite range vector fields merely differ from Maxwell's by the addition of a term

$$
I_{m}(A)=\frac{m^{2}}{2} \int d t d^{d} x\left[A_{0}^{2}-\mathbf{A}^{2}\right],
$$

resulting in the shift from the infinite range Coulomb to a (still repulsive) Yukawa interaction. Even the sign of $m^{2}$ is fixed by physics: changing it results in tachyonic propagation of the field excitations, and the relative sign between $A_{0}^{2}$ and $\mathbf{A}^{2}$ is forced by Lorentz covariance, $\mathbf{A}^{2}-A_{0}^{2}=A_{\mu} A^{\mu}$, in terms of the four-vector potential $A_{\mu}$. Thus, even if electrodynamics had a finite range, our sign conclusions would be unaffected.

\section{GRAVITY}

We turn now to our other main subject, gravity. The reduction to the Newtonian limit of full general relativity is rather complicated; even the notion of static limit must be analyzed carefully, because in this theory with space-time coordinate invariance, "static" means with respect to an "inertial" frame. Furthermore, the Newtonian limit (see, for example, Ref. 2) involves weak slowly moving sources or large separations between heavy ones. Nevertheless, the physical upshot is effectively that (after these tricky safeguards are understood) the force is governed by the weak gravity limit, namely the linear massless spin 2 field. We therefore turn to the latter, starting with its action and field equations in terms of the linearized Einstein tensor $G_{\mu \nu}^{L}$ :

$$
\begin{aligned}
I_{2}\left[h_{\mu \nu}\right. & \left.; T^{\mu \nu}\right]=\int d t d^{d} x\left[h_{\mu \nu} G_{L}^{\mu \nu}(h)+\kappa T^{\mu \nu} h_{\mu \nu}\right], \\
2 G_{\mu \nu}^{L} \equiv & \square h_{\mu \nu}-\left(\partial_{\alpha \nu}^{2} h_{\mu}^{\alpha}+\partial_{\alpha \mu}^{2} h_{\nu}^{\alpha}\right)+\partial_{\mu \nu}^{2} h_{\alpha}^{\alpha}-\eta_{\mu \nu}\left(\square h_{\alpha}^{\alpha}\right. \\
& \left.-\partial_{\alpha \beta}^{2} h^{\alpha \beta}\right)=-\kappa T_{\mu \nu} .
\end{aligned}
$$

The overall sign of $I_{2}$ yields the $+\frac{1}{2} \int \dot{h}_{i j}^{2}$ leading graviton kinetic term. By Eq. (12), the Bianchi identity, $\partial_{\mu} G_{L}^{\mu \nu} \equiv 0$, forces conservation of $T^{\mu \nu}$. [It also is easy to verify the action's gauge invariance under $\delta h_{\mu \nu}=\partial_{\mu} \xi_{\nu}+\partial_{\nu} \xi_{\mu}$, because also $G_{L}^{\mu \nu}(h+\delta h) \equiv G_{L}^{\mu \nu}(h)$.]

If we first follow the static limit approach, only two fields, $h_{00}$ and $\nabla^{2} h^{T} \equiv \frac{1}{2}\left(\delta_{i j} \nabla^{2}-\partial_{i j}^{2}\right) h_{i j}$ are relevant; the former is the counterpart of $A_{0}$, and the latter plays the role of $\mathcal{E}$ and is (like $\mathcal{E})$ gauge-invariant. In this static limit approach, which (as for Maxwell) is a gauge-dependent procedure, it is a straightforward consequence of Eqs. (11) and (12) that 


$$
\begin{aligned}
I_{2}\left[h_{00} ; T_{00}\right] & \rightarrow \int d t d^{d} x\left[h_{00}\left(\kappa T_{00}+\nabla^{2} h^{T}\right)-\frac{1}{4} h^{T} \nabla^{2} h^{T}\right] \\
& \rightarrow-\frac{1}{4} \kappa^{2} \int d t d^{d} x T_{00} G T_{00} .
\end{aligned}
$$

Here and henceforth, we specialize to our $d=3$ world; the generic $d$ dependence is given in Appendix B. The first integral shows (in the "Coulomb" gauge) the action's reduced field dependence for weak static $T^{00}$; the second, its form upon eliminating the "Newton" constraint $\nabla^{2} h^{T}+\kappa T_{00}=0$. Attraction between like, that is, positive mass, particles follows irrespective of the sign of $\kappa$. [See Ref. 3 for some amusing generalizations.] The magnitude of $\kappa^{2}$ is twice that of the Newtonian constant, as defined nonrelativistically in Eq. (3).

We now come to the more refined treatment, where gauge invariance is maintained and no static limit is required. The linearized action (14) is first expressed with space and time components (as well as orthogonal components of $h_{0 i}$ ) separated,

$$
\begin{aligned}
I_{2}\left[h_{i j}, h_{0 i} \equiv\right. & \left.N_{i}^{T}+N_{i}^{L}, h_{00} \equiv N ; T^{i j}, T^{0 i}, T^{00}\right] \\
\equiv & \int d t d^{d} x h_{\mu \nu} G_{L}^{\mu \nu}=\frac{1}{2} \int d t d^{d} x\left\{\left[h_{i j} \square h_{i j}\right.\right. \\
& -h_{i i} \square h_{j j}+2 N \square h_{i i}-2 N_{i}^{T} \nabla^{2} N_{i}^{T}-2 N h_{i j, i j} \\
& +2 h_{i i}\left(h_{\ell m, \ell m}-2 \dot{N}_{i, i}+\ddot{N}\right)-4 h_{i j, j} \dot{N}_{i} \\
& \left.\left.+2\left(h_{i j, j}\right)^{2}\right]+2 \kappa\left(h_{i j} T^{i j}+2 N_{i} T^{0 i}+N T^{00}\right)\right\} ;
\end{aligned}
$$

commas denote partial derivatives. We first retrace the static limit results, keeping only the dependence on the relevant variables:

$$
\begin{aligned}
I_{L}\left[h_{i j}, N, \partial_{t}=\right. & 0] \rightarrow \frac{1}{2} \int d t d^{d} x\left[h_{i j} \nabla^{2} h_{i j}-h_{i i} \nabla^{2} h_{j j}\right. \\
& +2 N \nabla^{2} h_{i i}-2 N h_{i j, i j}+2 h_{i i} h_{\ell m, \ell m} \\
& \left.+2 \kappa N T^{00}\right] .
\end{aligned}
$$

The part of Eq. (15) involving $T^{00}, N$, and $\nabla^{2} h^{T}$ correctly reduces to Eq. (13),

$$
\begin{aligned}
I_{L}\left[h^{T}, N, T^{00}\right] \rightarrow & \int d t d^{d} x\left[N\left(\kappa T^{00}+\nabla^{2} h^{T}\right)\right. \\
& \left.-\frac{1}{4} h^{T} \nabla^{2} h^{T}\right] .
\end{aligned}
$$

However, although $\nabla^{2} h^{T}$, being the component $G_{00}^{L}$ of the (gauge invariant) linear Einstein tensor $G_{\mu \nu}^{L}$ also is invariant, this reduction process does involve gauge choices by assuming various gauge components of the metric to be timeindependent.

We will now indicate how to bypass these assumptions as well as time-independence itself. Before doing so, we mention that something else has been (usefully) bypassed here and by the next procedure. We are obtaining the two-particle interaction term directly, thereby avoiding the apparent textbook paradox that a slowly moving particle's geodesic equation $\ddot{\mathbf{r}} \cong \frac{1}{2} \boldsymbol{\nabla} h_{00}$, whereas it is the gauge invariant component
$h^{T}$ that ought to be the Poisson equation potential according to Eq. (16). The equivalence of $h_{00} \equiv N$ and $h^{T}$ can obviously only be valid in certain "static" gauges. ${ }^{2}$

To formulate the relevant part of Eq. (14) in terms of gauge invariants only, we begin by noting that the use of stress tensor conservation, $\partial_{\mu} T^{\mu \nu}=0$ (the linearized approximation is in any case valid only for prescribed, conserved, sources) enables us to rewrite the interaction term as:

$$
\begin{aligned}
& \kappa \int d t d^{d} x h_{\mu \nu} T^{\mu \nu}=\kappa \int d t d^{d} x \psi T^{00}, \\
& \nabla^{4} \psi \equiv \nabla^{4} N-2 \nabla^{2} \dot{N}_{i, i}+\ddot{h}_{i j, i j} \equiv \nabla^{2} R_{00}-G_{i j, i j} .
\end{aligned}
$$

Because $\psi$ is a combination of (intrinsically gauge-invariant) curvature components, its gauge invariance is guaranteed. We now look for the other terms in Eq. (14) that depend on $N($ or $\psi)$, that is, the combination $\psi \nabla^{2} h^{T}$. Finally, we find the remaining dependence of Eq. (14) on $h^{T}$ which is the covariantized version of the static, $\int h^{T} \nabla^{2} h^{T}$, combination of Eq. (16), after setting $\nabla^{2} \rightarrow \square$. So the relevant gauge invariant, but nonstatic, part of Eq. (14) reduces to

$$
\begin{aligned}
I_{L}\left[\psi, h^{T}, T^{00}\right]= & \int d t d^{d} x\left\{\psi\left[\kappa T^{00}+\nabla^{2} h^{T}\right]-\frac{1}{4} h^{T} \square h^{T}\right\} \\
= & -\frac{\kappa^{2}}{2} \int d t d^{d} x\left\{T^{00} G T^{00}\right. \\
& \left.-T^{00} G G \partial_{0}^{2} T^{00}\right\},
\end{aligned}
$$

upon using $\square \equiv \nabla^{2}-\partial_{0}^{2} \equiv G^{-1}-\partial_{0}^{2}$ and eliminating the nowfamiliar constraint.

At first sight, Eq. (18) would seem to embody a retarded version of the Newtonian law, but in fact we can remove the retardation: the $\int T^{00} G G \partial_{0}^{2} T^{00}$ term can be converted into an instantaneous momentum interaction, using conservation, $\dot{T}^{00}+\partial_{i} T^{0 i}=0$, to remove the time derivatives. Then $\int \dot{T}^{00} G G \dot{T}^{00}=\int \partial_{i} T^{0 i} G G \partial_{j} T^{0 j}=-\int T_{L}^{0 i} G T_{L}^{0 i}$, where the vector $T_{L}^{0 i}$ is the longitudinal momentum density. Because this is a tensor theory, there are now both $T^{00}-T^{00}$ and $T^{0 i}-T^{0 i}$ instantaneous interactions. For slow particles, $T^{0 i}=0$, and only the Newtonian force survives.

We have provided a gauge and Lorentz invariant treatment of weak gravity that yields (without taking explicit static limits) precisely the instantaneous Newtonian force law between energy densities. As in electrodynamics, manifest Lorentz invariance has been given up for this privilege. Appendix $B$ reassures us that it is not really lost.

\section{SUMMARY}

That Coulomb and Newtonian forces are subsumed in their relativistic Maxwell and Einstein extensions is a truism. We have tried to exhibit some of these theories' qualitative triumphs based on this truism: The signs of their static, nonrelativistic forces are not only fixed (and the total charges and masses necessarily constant), but correlated to the (observationally verified) stability of the fundamental, ultrarelativistic, free field radiation, namely the (classical) photons and gravitons. That is, we related the static forces' signs to those of the free lightlike excitations that do not even couple to static sources: Despite their qualitatively different roles, the static and dynamic field components are linked kinemati- 
cally by being part of a single (vector or tensor) Lorentz entity, and the corresponding static force signs are correlated to the (odd or even) spins of the fields.

\section{ACKNOWLEDGMENTS}

I thank F. Ravndal for insisting on the pedagogical interest of this ancient lore (updated to include form fields), and $\mathrm{J}$. Franklin for comments. This research was supported by NSF Grant No PHY04-01667.

\section{APPENDIX A: FORMS AND HIGHER SPINS}

The Maxwell and Einstein actions have obvious extensions when we attach more indices to the basic fields: they can enter antisymmetrically-the so-called form fields-or symmetrically as in gravity's two-index metric field, not to mention fields of mixed symmetry.

We begin with form fields, whose current interest is due to their appearance in string theory. A form field has a totally antisymmetric potential $A_{[\mu \nu \ldots]}$, and associated field strength $B_{[\lambda \mu \nu \ldots]}=\partial_{[\lambda} A_{\mu \nu \ldots]}$ subject to the action

$$
I_{\text {form }}[A]=\int d t d^{d} x\left\{+\frac{1}{2} \dot{A}_{[i j \ldots]}^{2}+\cdots+J^{\mu \nu \cdots} A_{\mu \nu \ldots}\right\},
$$

which directly mimics Maxwell's action but with an antisymmetric current $J^{[\mu \nu \ldots]}$ (square brackets denote total antisymmetrization of included indices). Clearly, the only departure from Maxwell lies in the number of indices. Because there is still only one static source $\sim J^{0 i \ldots}$ coupled to $A_{0 i \ldots}$, and the spatial indices do not affect any signs upon being moved, $J^{0 i \ldots}=J^{0}{ }_{i \ldots}$, we can conclude that like static sources $J^{0 i \ldots}$ repel each other, just as in the "one-form," Maxwell case. (The one exception is the degenerate "zero-form," that is, the scalar, where there are no indices at all.)

The other main line extension beyond symmetric twotensors is to symmetric tensor fields, $h_{\mu \nu \alpha \ldots}$. These systems describe higher spin excitations, with spin values $s$, equal to the number of indices of $h_{\mu \nu \alpha \ldots}$. Here the essential-and to date only physical-application is to (spin 2) gravity. For all spins, the actions are of the form

$$
\begin{aligned}
I_{s \geqslant 2}\left[h_{\mu \nu \ldots} ; T^{\mu \nu \ldots]}\right]= & \frac{1}{2} \int d t d^{d} x\left\{\frac{1}{2} \dot{h}_{i j \ldots}^{2}+\cdots\right\} \\
& +\kappa \int d t d^{d} x T^{\mu \nu \cdots} h_{\mu \nu \ldots},
\end{aligned}
$$

where $T^{\mu \nu \ldots}$ is (necessarily) a symmetric tensor. We have omitted the additional terms in the free action required for gauge invariance, as well as a mass term that would appear in the finite range versions of Eq. (A2). Actually, elementary (as against composite) spin $>2$ fields are prone to coupling inconsistencies, have never been seen, and conserved dynamical (in contrast to fixed) higher rank symmetric sources $T^{\mu \nu \alpha \ldots}$ are physically excluded. ${ }^{4}$ Apart from these little problems, the alternation of signs of the force with spin follows directly from Eq. (12): The overall sign of the free action is determined so that the propagating modes, $h_{i j \ldots}$, have kinetic terms $+\frac{1}{2} \int\left(\dot{h}_{i j \ldots}\right)^{2}$. This sign again fixes that of the "Newtonian" terms according to the number of time indices involved: even/odd $s$ implies attraction/repulsion, where $s$ simultaneously counts spin and number of indices, by exactly the same analysis as for $s=2 / 1$ in the text.

\section{APPENDIX B: A COVARIANT DERIVATION}

For the experts, we append a rapid covariant, but less detailed, derivation of our results. If one "completes the squares" in the covariant scalar (4), Maxwell (5), and Einstein (11) actions, using the respective propagators (in any gauge, because the sources are conserved), one obtains the standard expressions

$$
\begin{aligned}
I_{s}[\rho]= & -\frac{1}{2} \int d t d^{d} x \quad \rho \square^{-1} \rho \rightarrow-\frac{1}{2} \int \rho G \rho, \\
I_{M}[j]= & -\frac{1}{2} \int d t d^{d} x \quad j^{\mu} \square^{-1} j_{\mu} \rightarrow+\frac{1}{2} \int j^{0} G j^{0}, \\
I_{2}[T]= & -\frac{\kappa^{2}}{2} \int d t d^{d} x\left[T^{\mu \nu} \square^{-1} T_{\mu \nu}-(d\right. \\
& \left.-1)^{-1} T_{\mu}^{\mu} \square^{-1} T_{\nu}^{\nu}\right] \\
\rightarrow & -\frac{1}{2}\left(\frac{d-2}{d-1}\right) \kappa^{2} \int T^{00} G T^{00},
\end{aligned}
$$

for the effective interactions and their static limits. Here $\square^{-1}$ is (say) the retarded propagator, whose static limit is our $G$. The overall signs of all actions are identical, as befits the fact that they come from the $\frac{1}{2} \int \phi \square \phi, \quad \frac{1}{2} \int A^{\mu} \square A_{\mu}$ and $\frac{1}{2} \int h_{\mu \nu} \square h^{\mu \nu}$ kinetic terms, with the same sign to ensure stable free excitations. Instead, the scalar/Coulomb/Newton sign difference is entirely encoded in the last terms, according to the number of zeros (equal to the number of the static source's indices or spin) to be raised or lowered.

The novel term in the tensor case is due to the fact that the graviton propagator involves a trace factor. The special values of $d$ arise as follows: There are no Newtonian forces in $d=2$ Einstein theory, ${ }^{5}$ while at $d=1$ the Einstein tensor vanishes identically, so Eqs. (11) and (12) become inconsistent.

\footnotetext{
${ }^{a}$ Electronic mail: deser@brandeis.edu

${ }^{1}$ For a recent discussion, see S. Deser and J. Franklin, "Schwarzschild and Birkhoff a la Weyl,’ Am. J. Phys. 73, 261-264 (2005); gr-qc/0408067.

${ }^{2}$ R. Arnowitt, S. Deser, and C. Misner, "The dynamics of general relativity," in Gravitation: An Introduction to Current Research, edited by L. Witten (Wiley, New York, 1962), pp. 227-265, reprinted in gr-qc/0405109.

${ }^{3}$ S. Deser and F. Pirani, "The sign of the gravitational force," Ann. Phys. 43, 436-451 (1967).

${ }^{4} \mathrm{~S}$. Weinberg, "Photons and gravitons in S-matrix theory: Derivation of charge conservation and equality of gravitational and inertial mass," Phys. Rev. 135, B1049-B1056 (1964); "Photons and gravitons in perturbation theory: Derivation of Maxwell's and Einstein's equations," 138, B988B1002 (1965).

${ }^{5}$ S. Deser, G. 't Hooft, and R. Jackiw, “Three-dimensional Einstein gravity: Dynamics of flat space," Ann. Phys. 152, 220-235 (1984).
} 